\title{
Point particles in $2+1$ dimensions: toward a semiclassical loop gravity formulation
}

\author{
Jonathan Ziprick \\ Perimeter Institute and University of Waterloo, \\ Waterloo, Ontario, Canada
}

June 22, 2021

\begin{abstract}
We study point particles in $2+1$ dimensional first order gravity using a triangulation to fix the connection and frame-field. The Hamiltonian is reduced to a boundary term which yields the total mass. The triangulation is dynamical with non-trivial transitions occurring when a particle meets an edge. This framework facilitates a description in terms of the loop gravity phase space.
\end{abstract}

\section{Introduction}

Three dimensional gravity has often been used as a toy model for the four dimensional theory. Here we study point particles in $2+1$ dimensions using spatial geometries analogous to those in 11. These geometries are isomorphic to a gauge-reduced holonomyflux phase space which descends from the $\hbar \rightarrow 0$ limit of a loop quantum gravity Hilbert space.

We consider a Hamiltonian written in terms of frame-field and connection variables. The spacetime signature is taken to be Euclidean since the relevant gauge group is then $\mathrm{SU}(2)$, as in $3+1$ dimensions with Lorentzian signature. Our first step is to specify the variables over an entire spacelike slice using a triangulation where particles sit on the vertices. After gauge fixing, the Hamiltonian is reduced to a boundary term which equals the total particle mass. In this gauge the triangulation evolves according to particle momenta and undergoes discrete changes when a par- ticle meets an edge, collapsing one of the triangles.

\section{Hamiltonian formulation}

Spacetime is taken to be $M=\mathbb{R} \times \Sigma$ where $\Sigma$ is a spacelike surface homeomorphic to a disc. The first order formalism of general relativity parameterizes the gravitational field in terms of a connection $\mathbf{A}$ and a frame-field $\mathbf{e}$, both being one-forms on $\Sigma$ taking values in the $\mathfrak{s u}(2)$ algebra. We use $\mathfrak{s u}(2)$ basis elements $\tau^{i}$ (for $i=0,1,2$ ) which are given by $-i / 2$ times the Pauli matrices. Our notation is such that elements of $\mathfrak{s u}(2)$ are written as $\mathbf{A} \equiv A^{i} \tau^{i}$, for example, and all internal indices are written as superscripts.

In units where $8 \pi G=c=1$ a Hamiltonian for pure gravity is given by [2]:

$$
\begin{aligned}
H & =-\int_{\Sigma}\left(N^{i} F^{i}+\lambda^{i} G^{i}\right)+\int_{\partial \Sigma} N^{i} A^{i}, \\
F^{i} & =\mathrm{d} A^{i}+\frac{1}{2} \epsilon^{i j k} A^{j} \wedge A^{k} \\
G^{i} & =\mathrm{d} e^{i}+\epsilon^{i j k} A^{j} \wedge e^{k} .
\end{aligned}
$$

$N^{i}$ and $\lambda^{i}$ are Lagrange multipliers for the flatness and Gauss constraints respectively. We normalize the time coordinate by choosing $|N|^{2}=1$. The boundary term and the condition $\left.\lambda^{i}\right|_{\partial \Sigma}=0$ ensure that the variational principal is well-defined and the constraints are first class. The Poisson algebra is:

$$
\left\{A_{a}^{i}(x), e_{b}^{j}(y)\right\}=\epsilon_{a b} \delta^{i j} \delta^{2}(x-y),
$$


where $a=1,2$ labels the space coordinates.

It is well-known that in $2+1$ dimensions point particles represent conical singularities 3. We introduce particles by replacing the flatness constraint with $F^{i}-\sum_{I} \delta^{2}\left(x-q_{\pi}\right) p_{\pi}^{i} \mathrm{~d} x^{2}$ so that each particle $\pi$ gives a contribution to the curvature proportional to its momentum $\mathbf{p}_{\pi}$ at the location $x=q_{\pi}[2]$. In this treatment the particles are defined by the fields $(\mathbf{A}, \mathbf{e})$ and do not have independent parameters of their own. We consider the total mass to be less than $2 \pi$ so that $\Sigma$ is open 3 .

\section{Gauge fixing}

In order to solve the constraints and reduce the Hamiltonian, we triangulate $\Sigma$ according to the particle locations so that we may give a piecewise definition of $(\mathbf{A}, \mathbf{e})$ on $\Sigma \backslash\left\{q_{\pi}\right\}$. See [4] for other approaches along these lines. For clarity, we take the boundary $\partial \Sigma$ to be triangular; the generalization to arbitrary polygons follows simply. We take each particle position to be an internal vertex and connect all vertices with straight edges to obtain a triangulation. There is ambiguity in this procedure, but the number of triangles $T$ is fixed by the number of particles $\Pi$ to be $T=2(\Pi+1)-1$ via the Euler characteristic for planar graphs. Different triangulations generally lead to different gauge choices.

Within each triangle $\Delta$, we must choose $\left(\mathbf{A}_{\Delta}, \mathbf{e}_{\Delta}\right)$ such that $G^{i}=F^{i}=0$. The general solution [1] is given by an $\mathrm{SU}(2)$ group element $a_{\Delta}$ and a closed one-form $\chi_{\Delta}$ :

$$
\mathbf{A}_{\Delta}=a_{\Delta} \mathrm{d} a_{\Delta}^{-1}, \quad \mathbf{e}_{\Delta}=a_{\Delta} \chi_{\Delta} a_{\Delta}^{-1} .
$$

Consider two adjacent triangles labeled 1 and 2. We ensure that the fields are continuous across the shared edge $e$ by requiring that there exists an $h_{e} \in \mathrm{SU}(2)$ on the edge such that:

$$
\left.a_{2}\right|_{e}=\left.a_{1} h_{e}\right|_{e},\left.\quad \chi_{2}\right|_{e}=\left.h_{e}^{-1} \chi_{1} h_{e}\right|_{e} .
$$

Now, if we integrate the curvature over a region containing a single vertex $\pi$ we must obtain $\int F^{i}=$ $p_{\pi}^{i}$. In order to specify the group elements $a_{\Delta}$ we subdivide each triangle into three regions defined by edges joining the centroid to the vertices. Consider one such region $\Delta_{r}$ where points $\left(0, \pm y_{0}\right)$ are the endpoints of an edge, and $\left(x_{0}, y_{1}\right)$ is the centroid of the triangle. We define:

$$
u=\tanh ^{-1}\left(v_{+}\right)+\tanh ^{-1}\left(v_{-}\right)
$$

where $v_{ \pm}=\frac{x}{x_{0}} \pm\left(\frac{y}{y_{0}}-\frac{x y_{1}}{x_{0} y_{0}}\right)$. Note that the line $u=0$ coincides with the edge, and $u=\infty$ consists of lines between the centroid of the triangle and the endpoints of the edge. We set the group element in a region equal to:

$$
a_{\Delta_{r}}(u)=\exp \left(\mathbf{P} \int_{0}^{u} f(\tilde{u}) \mathrm{d} \tilde{u}\right)
$$

where we have introduced a bump function $f(u)$ which satisfies $\int_{0}^{\infty} f(u) \mathrm{d} u=1$. For a given $u$, this group element defines a rotation about a direction $\mathbf{P} /|P|$ by an angle proportional to $|P|$.

With these ingredients it is now possible to choose a gauge, i.e. to specify the frame-field and connection on $\Sigma \backslash\left\{q_{\pi}\right\}$. Given a set of particles $\left\{q_{\pi}, p_{\pi}\right\}$, one first defines a boundary and introduces a triangulation. Then we arbitrarily choose a triangle (labeled 1) and make a choice for $\left(a_{1}, \chi_{1}\right)$. Moving to a neighbouring triangle, we specify $a_{2}$ here and use 4 to find $\chi_{2}$. We continue in this manner until $\left(a_{\Delta}, \chi_{\Delta}\right)$ (and thereby $\left.\left(\mathbf{A}_{\Delta}, \mathbf{e}_{\Delta}\right)\right)$ are defined in each triangle, taking care to ensure the connection yields proper curvature at each vertex by choosing the parameter $\mathbf{P}$ in each $a_{\Delta_{r}}$ to be consistent with particle momenta. The aforementioned relationship between the number of triangles and the number of particles ensures that this procedure will work for arbitrarily many particles. See 5 for a more detailed description of this process.

Preserving the gauge dynamically leads to a condition on the Lagrange multipliers:

$$
\mathrm{d}_{A} N^{i}+\epsilon^{i j k} e^{j} \lambda^{k}=0 .
$$

This fixes three of the six degrees of freedom in $N^{i}$ and $\lambda^{i}$. We eliminate the remaining ambiguity by choosing $\mathrm{d}_{A} N^{i}=0$ and setting $\mathbf{N}\left(q_{\pi}\right)=\mathbf{p}_{\pi} / m$. This choice ensures that particles move along with the triangulation and remain at the vertices. 


\section{Dynamics and observables}

After specifying $(\mathbf{A}, \mathbf{e})$, the Hamiltonian is reduced to a boundary term which evaluates to:

$$
\begin{aligned}
H & =\int_{\partial \Sigma} N^{i} A^{i}=\int_{\Sigma} N^{i} \mathrm{~d}_{A} A^{i} \\
& =\sum_{\pi} N^{i}\left(q_{\pi}\right) p_{\pi}^{i}=\sum_{\pi} m_{\pi},
\end{aligned}
$$

where we used Stokes' theorem, the conditions on $\mathbf{N}$, and the flatness constraint.

Dynamics are implicitly determined by the direction of momentum $N^{i}\left(q_{\pi}\right)$ for each particle via the equation of motion:

$$
\dot{q}_{\pi}^{i}(t)=q_{\pi}^{i}(0)+t N^{i}\left(q_{\pi}\right),
$$

and the triangulation evolves accordingly. Nontrivial, discrete transitions occur when a particle meets an edge. Consider triangles 1 and 2 depicted in fig. 1. If the particle at the top of the left hand

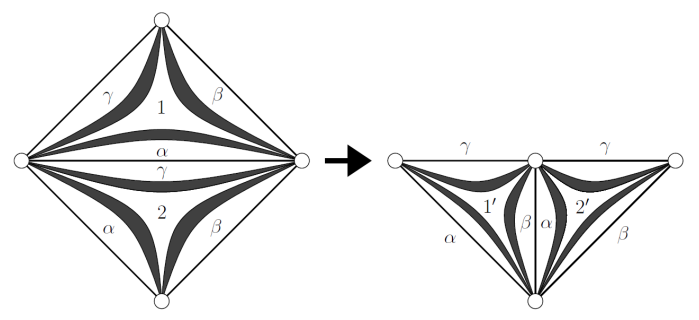

Figure 1: Before and after a discrete change in triangulation. $\alpha, \beta, \gamma$ label the three regions of each triangle. Grey regions (bounded by constant- $u$ lines) indicate where the connection is generally non-zero.

side of the figure moves downward until it reaches the shared edge, then 1 closes and 2 splits into $1^{\prime}$ and $2^{\prime}$ (shown on the right hand side) preserving the total number of triangles. The fields are defined in the new triangles by specifying the $\mathrm{SU}(2)$ rotation parameters:

$$
\begin{array}{ll}
\mathbf{P}_{1_{\gamma}^{\prime}}=\mathbf{P}_{1_{\alpha}}+\mathbf{P}_{2_{\gamma}}+\mathbf{P}_{1_{\gamma}}, & \mathbf{P}_{1_{\alpha}^{\prime}}=\mathbf{P}_{2_{\alpha}}, \\
\mathbf{P}_{2_{\gamma}^{\prime}}=\mathbf{P}_{1_{\alpha}}+\mathbf{P}_{2_{\gamma}}+\mathbf{P}_{1_{\beta}}, & \mathbf{P}_{2_{\beta}^{\prime}}=\mathbf{P}_{2_{\beta}}, \\
\mathbf{P}_{1_{\beta}^{\prime}}=\mathbf{P}_{2_{\alpha}^{\prime}}=0 . &
\end{array}
$$

Notice $h_{e}=\mathbb{1}$ along the new edge shared by $1^{\prime}$ and $2^{\prime}$ so that $\chi_{1^{\prime}}=\chi_{2^{\prime}}=\chi_{2}$. The fields in all other triangles are unaffected by this transition.

\section{Conclusion}

We have described a system of point particles in $2+1$ dimensional gravity in terms of evolving triangulations. A gauge choice for $(\mathbf{A}, \mathbf{e})$ was specified by choosing $a_{\Delta}$ and $\chi_{\Delta}$ in each triangle. The triangulation evolves according to particle dynamics so that particles remain at vertices at all times. The discrete change in triangulation that occurs when a vertex meets an edge is well-defined.

This construction yields spatial geometries that are the two-dimensional analog of those in [1]. Having $\left(a_{\Delta}, \chi_{\Delta}\right)$ in each triangle allows us to immediately write this data in terms of holonomy-flux variables. Moreover, knowing how $\left(a_{\Delta}, \chi_{\Delta}\right)$ behave under a discrete change in triangulation tells us how the holonomies and fluxes will change. This sets the stage for a semiclassical loop gravity description of the system [5].

Acknowledgements: The author is grateful to Gabor Kunstatter, Laurent Freidel and John Moffat for helpful conversations during the course of this work.

\section{References}

[1] L. Freidel, M. Geiller and J. Ziprick, arXiv:1110.4833v1 [gr-qc] (2011).

[2] L. Freidel and D. Louapre, Class. Quant. Grav. 21, 5685 (2004).

[3] S. Deser, R. Jackiw and G. 't Hooft, Annals of Physics 152, Issue 1, 220 (1984).

[4] G. 't Hooft, Class. Quantum Grav. 91335 (1992); H.J. Matschull, Class. Quant. Grav. 18, 3497 (2001).

[5] J. Ziprick, in preparation. 